\title{
Isolated gallbladder perforation after trivial abdominal trauma in a child: a case report and review of the literature
}

\author{
Pradeep balmiki, Rajesh lonare, Ashwin apte, Nirpex Tyagi, Rajeev Singh \\ Department of General Surgery, People's College of Medical Sciences and Research Centre Bhopal, India
}

\begin{abstract}
Gallbladder injury after blunt abdominal trauma is rare. Isolated gall bladder perforation due to blunt abdominal trauma is even rarer. The clinical presentation of gallbladder injury is variable resulting in delay in diagnosis and treatment. Early diagnosis is thus essential but still remains a challenge. We present a case of 12 year old child with isolated gall bladder perforation following trivial blunt trauma to the abdomen.

Keywords: Abdominal trauma, Gallbladder, Paediatric, Perforation.
\end{abstract}

\section{Introduction}

The gallbladder is a well-protected organ, being partially embedded in the liver substance. It is protected by the liver, intestines, the omentum and ribs. Gallbladder injury is rarer after blunt abdominal trauma and usually associated with additional visceral injuries. Isolated gall bladder perforation by blunt abdominal injury is even rarer. It results in vague symptoms which often lead to delayed diagnosis and therapy. Early exploratory laparotomy is recommended to reduce the high morbidity associated with this condition.

\section{Case report}

A 12 year old boy was admitted to the emergency with complaint of pain in abdomen. There was history of blunt trauma to abdomen 24 hours back when he was try to bend over to a filled water PVC tank. On admission he was hemodynamically stable with following vitals heart rate 84/min, BP 110/70 mmhg, RR 20/min and he was afebrile. Physical examination reveals tenderness over right upper abdomen with hypoactive bowel sounds. Laboratory investigations were within normal limit. Chest and abdominal radiographs were normal with no signs of free air. An abdominal computed tomogram (CT scan) was performed which showed tiny focal disruption of gallbladder wall with significant amount of free fluid in hepatorenal pouch, both flanks and in pelvis.

Patient underwent urgent exploratory laparotomy which revealed perforated gallbladder of size $1 \mathrm{~cm}$ with free bile. No other abdominal injuries were noted. After abdominal wash cholesystectomy was performed and an abdominal drain was placed. Postoperative course was uneventful and patient was discharged on the $9^{\text {th }}$ postoperative day.

\section{Discussion}

The first specimen of a lacerated gallbladder from a blunt trauma was found in Guy's Museum in London and dates from $1388^{1}$. The first known case of someone surviving a gallbladder traumatic rupture was in $1898^{1}$. Blunt injuries to the gall bladder occurs rarely ranging from $1.9 \%$ as reported by penn ${ }^{2}$ to $2.1 \%$ in the series of patients examined by soderstrom et $\mathrm{al}^{3}$. The incidence of isolated damage to the gallbladder is even smaller, as shown in soderstrom's review where by only 5 out of 30 cases of gallbladder injuries were isolated. This was also demonstrated by wiener et $\mathrm{al}^{4}$.showing that only half of the cases of gallbladder injury were in isolation. Liver injury is present in up to $83 \%$ of cases of gallbladder trauma and the spleen, stomach, intestines, pancreas and kidney may also be injured ${ }^{3}$. Penn et al classified four major groups of gall bladder injury contusion, avulsion, laceration and traumatic cholecystitis ${ }^{2}$.

The majority of gallbladder injuries occur following motor vehicle accident, significant falls and direct blows on the abdomen ${ }^{5}$.

A thin walled normal gallbladder or a distended gallbladder following a meal is more prone to traumatic rupture than a diseased thick walled one. A high incidence of traumatic gallbladder is reported in alcohol intoxicated patients ${ }^{6}$. Losanoff and Kjossev ${ }^{7}$ describe a more detailed classification of blunt gallbladder injuries; according to their classification, our patient belongs to type 2 (rupture; Figure 3 and Table 1) 7 .

The delay in presentation of the injury is not unusual. Damage to a non-infected gallbladder can cause leakage of sterile bile into the abdomen. This in itself does not present acutely and such injuries can take up to six weeks to become apparent ${ }^{5}$. The majority of these cases will be diagnosed peri -operatively although a few cases have demonstrated gallbladder damage using pre operative computed tomography.

Para clinical examinations may not be contributive, peritoneal lavage is helpful only if the bile is fluid and too many false negative results were reported in the literature ${ }^{8}$.The MRI study of the gallbladder depends 
on its ability to take the contrast which can be disrupted in a trauma case. The ultrasound, CT, MRI may help to make the diagnosis in some rare cases. The biliary isotope scientigraphy combined with computed tomography, MRI, and ERCP currently allow early diagnosis ${ }^{8}$.

As in our case, the outcome is often good. No deaths have been reported in patients with isolated gallbladder perforation.

Laparoscopic cholecystectomy is advocated to be a safe and effective procedure in the diagnosis and management of traumatic gallbladder perforation ${ }^{9}$.

In our case however due to uncertainty of the diagnosis, an exploratory laparotomy was performed as the safest option.

\section{Conclusion}

Isolated gallbladder injury after blunt abdominal trauma is rare. It is associated with vague symptoms so early diagnosis is a challenging job. Cholecystectomy is the safest and effective procedure.

\section{References}

[1]. Theodoros E Pavlidis, Miltiadis A Lalountas, Kyriakos Psarras, Nikolaos G Symeonidis,Anastasios Tsitlakidis, Efstathios T Pavlidis, Konstantinos Ballas, Nikolaos Flaris, Georgios N Marakis and Athanassios K Sakantamis, Isolated complete avulsion of the gallbladder (near traumatic cholecystectomy): a case report and review of the literature, Journal of Medical Case Reports 2011, 5:392.

[2]. Penn I, injuries of the gallbladder, British journal of surgery $1962 ;(49) ; 636$

[3]. Soderstrom c.a, mekawa k, du priest rw jr, crowly ra, gallbladder injuries resulting from blunt abdominal trauma, ann. Surg. 1981 ;( 193); 60-66

[4]. Wiener I, Watson lc, walma fj, Perforation of the gallbladder due to blunt abdo.trauma, arch surg., 1982 ;( 117); 805-807

[5]. Bainbridge $\mathrm{j}$, shaaban $\mathrm{h}$, kenefick $\mathrm{n}$,Armstrong $\mathrm{cp}$, Delayed presentation of an isolated gallbladder rupture following blunt abdominal trauma: a case report, $\mathrm{j}$ med case reports $2007 ; 1(52) ; 294-295$

[6]. P. schachter, A. czerniak, E shemesh, I avigad, G lotan and I wolfstein ; case report isolated gallbladder rupter due to blunt abdo trauma, HPB surgery $1989 ;(1) ; 359-362$.

[7]. Losanoff JE, Kjossev KT: Complete traumatic avulsion of the gallbladder.

[8]. Int J Care Injured 1999, (30):365-368.

[9]. O ndour, $\mathrm{H}$ moustapha, $\mathrm{N}$ ndoye, $\mathrm{G}$ ngom, Isolated gallbladder perforation after blunt abdominal trauma in children a case report , The internet journal of pediatrics and neonatology 2012; vol. 14; (1);14394

[10]. Liess bd, awad zt, eubanks ws, laporoscopic cholecystectomy for isolated traumatic rupture of the gallbladder following blunt abdominal injury, j. laparoendosc adv surg tech a 2006(16); 623-625

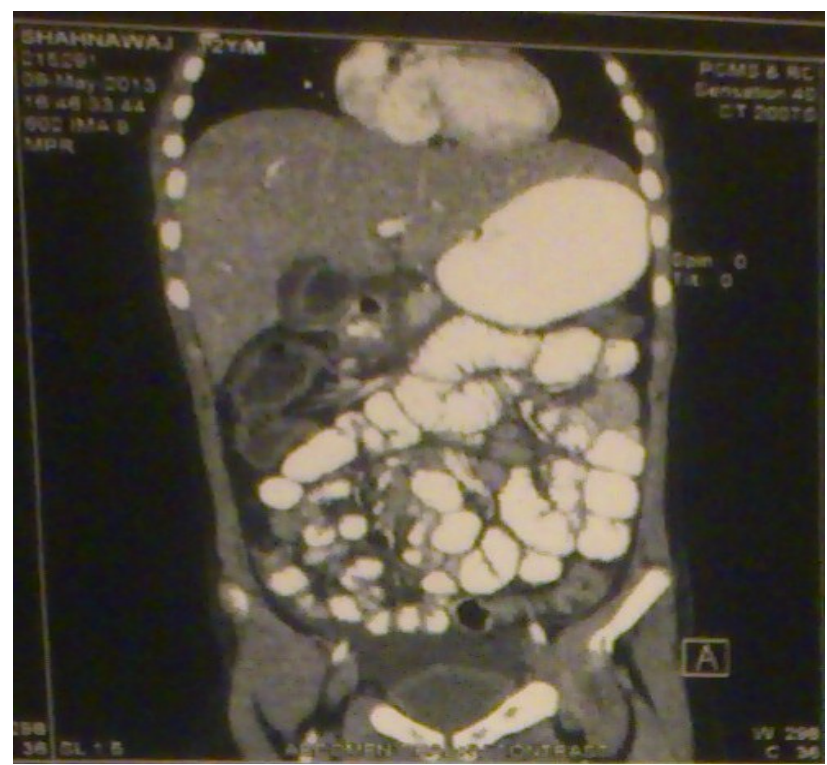

Fig 1: CT scan showing pericholecystic fluid 


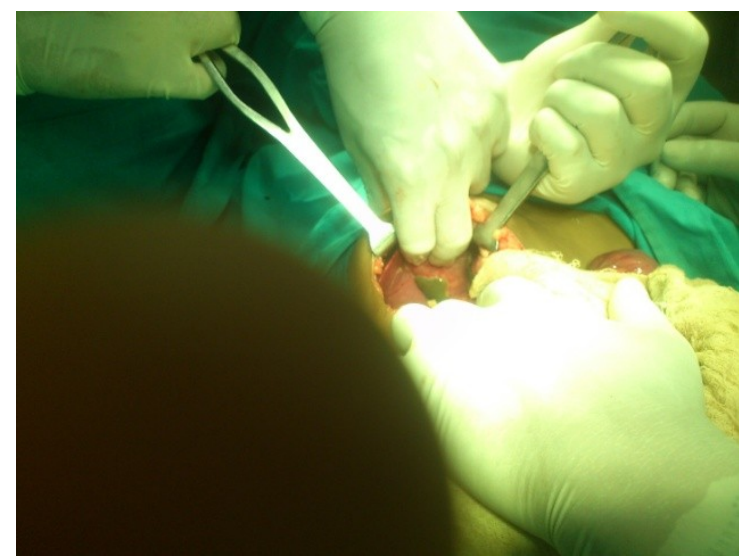

Fig 2: Picture showing Bile leak from gallbladder perforation

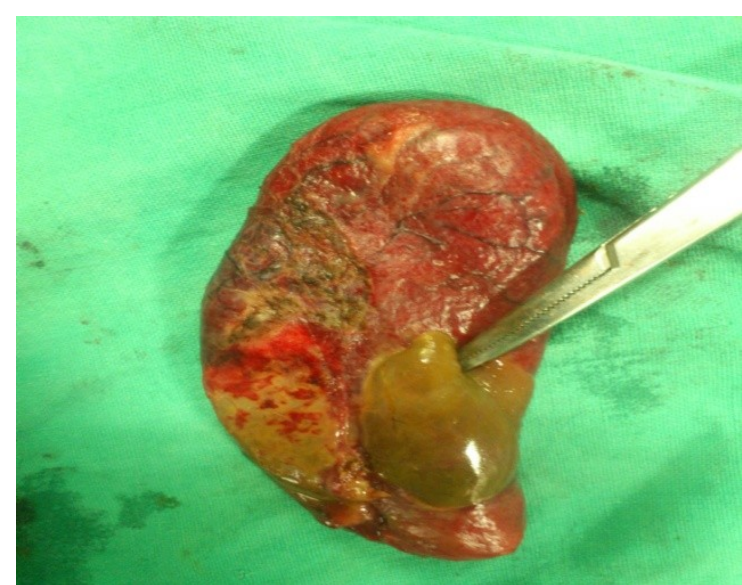

Fig 3: Picture showing gallbladder perforation

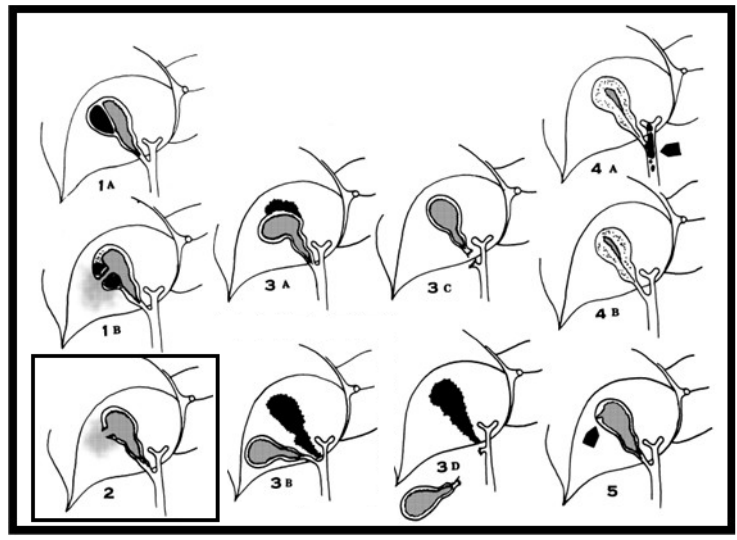

Fig 4: Schematic drawing of all known types of gallbladder injury according to the classification by Losanoff and $\mathrm{Kjossev}^{7}$. Our case is highlighted.

Table 1

Types of gallbladder injury according to the classification by Losanoff and Kjossev [4] (Figure 3)

Type

Injury of the gallbladder

1A Contusion with intramural hematoma

1B Contusion with perforation

2 Rupture

3A Avulsion with partial detachment

3B Avulsion with complete detachment from the liver but with attachment to the structures of the hepatoduodenal ligament (so-called "near traumatic cholecystectomy")

3C Torn only from the hepatoduodenal ligament 
3D Completely torn from all attachments (so-called "traumatic cholecystectomy")

4A Traumatic cholecystitis, secondary to hemobilia

4B Acute acalculus cholecystitis

5 Mucosal tear with leakage of bile 